\title{
The Role of Specialized Intelligent Body-System Networks in Guiding General-Purpose Cognition
}

\author{
Ben Goertzel
}

Novamente LLC

\begin{abstract}
Human cognition is portrayed as involving a highly flexible, self-organizing "cognitive network", closely coupled with a number of more specific intelligent "body-system networks" - e.g. those associated with the perceptual and motor systems, the heart, the digestive system, the liver, and the immune and endocrine systems, all of which have been shown to have their own adaptive intelligence. These specialized intelligent networks provide the general-purpose cognitive network with critical structural and dynamical inductive biasing. It is argued that early-stage AGI systems must involve roughly comparable inductive biasing, though not necessarily achieved in the same way.
\end{abstract}

\section{Introduction}

The most unique aspect of human intelligence is rooted in what one might call the "cognitive cortex" - the portions of the brain dealing with self-reflection and abstract thought. But the cognitive cortex does its work in close coordination with the body's various more specialized intelligent subsystems, including those associated with the gut, the heart, the liver, the immune and endocrine systems, and the perceptual and motor cortices.

In prior publications [1] we have argued that the human cognitive cortex or the core cognitive network of any roughly human-like AGI system - should be viewed as a highly flexible, self-organizing network. These cognitive networks are modelable e.g. as a recurrent neural net with general topology, or a weighted labeled hypergraph, and are centrally concerned with recognizing patterns in its environment and itself, especially patterns regarding the achievement of the system's goals in various appropriate contexts. Here we augment this perspective, noting that the human brain's cognitive network is closely coupled with a variety of simpler and more specialized intelligent "body-system networks" which provide it with structural and dynamical inductive biasing. We then discuss the implications of this observation for practical AGI design.

One recalls Pascal's famous quote "The heart has its reasons, of which reason knows not." As we now know, the intuitive sense that Pascal and so many others have expressed, that the heart and other body systems have their own reasons, is grounded in the fact that they actually do carry out simple forms of reasoning (i.e. intelligent, adaptive dynamics), in close, sometimes cognitively valuable, coordination with the central cognitive network. 


\section{Some of the Human Body's Specialized Intelligent Subsystems}

The human body contains multiple specialized intelligences apart from the cognitive cortex. Here we review some of the most critical.

Hierarchies of Visual and Auditory Perception . The hierarchical structure of visual and auditory cortex has been taken by some researchers [2], [3] as the generic structure of cognition. While we suspect this is overstated, we agree it is important that these cortices nudge large portions of the cognitive cortex to assume an approximately hierarchical structure.

Olfactory Attractors . The process of recognizing a familiar smell is grounded in a neural process similar to convergence to an attractor in a nonlinear dynamical system [4]. There is evidence that the mammalian cognitive cortex evolved in close coordination with the olfactory cortex [5], and much of abstract cognition reflects a similar dynamic of gradually coming to a conclusion based on what initially "smells right."

Physical and Cognitive Action . The cerebellum, a specially structured brain subsystem which controls motor movements, has for some time been understood to also have involvement in attention, executive control, language, working memory, learning, pain, emotion, and addiction [6].

The Second Brain . The gastrointestinal neural net contains millions of neurons and is capable of operating independently of the brain. It modulates stress response and other aspects of emotion and motivation based on experience resulting in so-called "gut feelings" [7].

The Heart's Neural Network. The heart has its own neural network, which modulates stress response, energy level and relaxation/excitement (factors key to motivation and emotion) based on experience [8].

Pattern Recognition and Memory in the Liver. The liver is a complex pattern recognition system, adapting via experience to better identify toxins [9]. Like the heart, it seems to store some episodic memories as well, resulting in liver transplant recipients sometimes acquiring the tastes in music or sports of the donor [10].

Immune Intelligence . The immune network is a highly complex, adaptive selforganizing system, which ongoingly solves the learning problem of identifying antigens and distinguishing them from the body system [11]. As immune function is highly energetically costly, stress response involves subtle modulation of the energy allocation to immune function, which involves communication between neural and immune networks. 
The Endocrine System: A Key Bridge Between Mind and Body. The endocrine (hormonal) system regulates (and is related by) emotion, thus guiding all aspects of intelligence (due to the close connection of emotion and motivation) [12].

Breathing Guides Thinking. As oxygenation of the brain plays a key role in the spread of neural activity, the flow of breath is a key driver of cognition. Forced alternate nostril breathing has been shown to significantly affect cognition via balancing activity of the two brain hemispheres [13].

Much remains unknown, and the totality of feedback loops between the human cognitive cortex and the various specialized intelligences operative throughout the human body, has not yet been thoroughly charted.

\section{Implications for AGI}

What lesson should the AGI developer draw from all this? The particularities of the human mind/body should not be taken as general requirements for general intelligence. However, it is worth remembering just how difficult is the computational problem of learning, based on experiential feedback alone, the right way to achieve the complex goal of controlling a system with general intelligence at the human level or beyond. To solve this problem without some sort of strong inductive biasing may require massively more experience than young humans obtain.

Appropriate inductive bias may be embedded in an AGI system in many different ways. Some AGI designers have sought to embed it very explicitly, e.g. with hand-coded declarative knowledge as in Cyc, SOAR and other "GOFAI" type systems. On the other hand, the human brain receives its inductive bias much more subtly and implicitly, both via the specifics of the initial structure of the cognitive cortex, and via ongoing coupling of the cognitive cortex with other systems possessing more focused types of intelligence and more specific structures and/or dynamics.

In building an AGI system, one has four choices, very broadly speaking:

1. Create a flexible mind-network, as unbiased as feasible, and attempt to have it learn how to achieve its goals via experience

2. Closely emulate key aspects of the human body along with the human mind

3. Imitate the human mind-body, conceptually if not in detail, and create a number of structurally and dynamically simpler intelligent systems closely and appropriately coupled to the abstract cognitive mind-network, provide useful inductive bias.

4. Find some other, creative way to guide and probabilistically constrain one's AGI system's mind-network, providing inductive bias appropriate to the tasks at hand, without emulating even conceptually the way the human mind-brain receives its inductive bias via coupling with simpler intelligent systems. 
Our suspicion is that the first option will not be viable. On the other hand, to do the second option would require more knowledge of the human body than biology currently possesses. This leaves the third and fourth options, both of which seem viable to us.

The OpenCog project [14], that is the main focus of our current AGI efforts, incorporates a combination of the third and fourth options. OpenCog's generic dynamic knowledge store, the Atomspace, is coupled with specialized hierarchical networks (DeSTIN) for vision and audition, somewhat mirroring the human cortex. An artificial endocrine system for OpenCog is also under development, as part of a project using OpenCog to control humanoid robots. On the other hand, OpenCog has no gastrointestinal nor cardiological nervous system, and the stress-response-based guidance provided to the human brain by a combination of the heart, gut, immune system and other body systems, is achieved in OpenCog in a more explicit way using the OpenPsi model of motivated cognition, and its integration with the system's attention allocation dynamics.

Likely there is no single correct way to incorporate the lessons of intelligent human body-system networks into AGI designs. But these are aspects of human cognition that all AGI researchers should be aware of.

\section{References}

1. Goertzel, B.: Patterns, hypergraphs and general intelligence. In: Proceedings of IJCNN 2006. (2009)

2. Kurzweil, R.: How to Create a Mind. Viking (2012)

3. Hawkins, J., Blakeslee, S.: On Intelligence. Brown Walker (2006)

4. Freeman, W.: Societies of Brains. Erlbaum (1995)

5. Rowe, T.: Fossil evidence on origin of the mammalian brain. Science 20 (2011)

6. Peter Strick, R.D., Fiez, J.: Cerebellum and nonmotor function. Annual Review of Neuroscience Vol. 32: 413-434 (2009)

7. Gershon, M.: The Second Brain. Harper (1999)

8. Armour, J.A.: Cardiac neuronal hierarchy in health and disease. Am J Physiol Regul Integr Comp Physiol 287: (2004)

9. Conolly, R., Blancato, J.: Computational modeling of the liver. NCCT BOSC Review (2006) http://www.epa.gov/ncct/bosc_review/2006/files/07_Conolly_ Liver_Model.pdf.

10. Effective-Mind-Control.com: Cellular memory in organ transplants. Effective Mind Control, (2012) http://www.effective-mind-control.com/ cellular-memory-in-organ-transplants.html, updated Feb 12012.

11. Farmer, D., Perelson, A.: The immune system, adaptation and machine learning. Physica D, v. 2 (1986)

12. Pang, Z., Han, W.: Regulation of synaptic functions in central nervous system by endocrine hormones and the maintenance of energy homeostasis. Bioscience Reports (2012)

13. Shannahoff-Khalsa, D., Boyle, M., Buebel, M.: The effects of unilateral forced nostril breathing on cognition. Int J Neurosci. (1991)

14. Goertzel, B., et al: The cogprime architecture for embodied artificial general intelligence. In: Proceedings of IEEE Symposium on Human-Level AI, Singapore. (2013) 